\title{
Knee stability, athletic performance and sport- specific tasks in non-professional soccer players after ACL reconstruction: comparing trans-tibial and antero-medial portal techniques
}

\author{
Cosimo Tudisco ${ }^{1}$ \\ Salvatore Bisicchia ${ }^{1}$ \\ Andrea Cosentino ${ }^{1}$ \\ Federica Chiozzi ${ }^{2}$ \\ Massimo Piva ${ }^{2}$ \\ 1 Department of Orthopaedic Surgery, University of \\ Rome Tor Vergata, Italy \\ 2 Isokinetic Medical Group, Rome, Italy
}

Corresponding author:

Cosimo Tudisco

Department of Orthopaedic Surgery, University of

Rome Tor Vergata

Viale Oxford 81

00133 Rome, Italy

E-mail: cosimo.tudisco@uniroma2.it

\section{Summary}

Background: a wrong position of bone tunnels, in particular on the femur, is one of the most frequent causes of a failed anterior cruciate ligament ( $A C L$ ) reconstruction. Several studies demonstrated that drilling the femoral tunnel through the antero-medial portal (AMP) allows a more anatomical placement on the lateral femoral condyle and higher knee stability, compared to trans-tibial (TT) technique. The aim of this study was to retrospectively evaluate two groups of soccer players operated on for ACL reconstruction according to either one of these two techniques.

Methods: two groups of non-professional soccer players operated on for a single bundle ACL reconstruction with hamstrings autograft using either a TT (20 patients) or an AMP (23 patients) technique were retrospectively evaluated with KT1000 arthrometer, manual pivot shift test, isokinetic test, the incremental treadmill-running test, athletic and sport specific tasks, and knee scores (IKDC, Lysholm and KOOS).

Results: the AMP group showed better results at pivot shift test and KOOS, but lower flexion angles at single leg squat test. There were no differences in all the other considered outcomes.

Conclusions: the better rotational stability of the knee achieved in AMP group did not lead to sig- nificantly better clinical and functional results in our patients.

Level of evidence III. Treatment study: Case-control study.

KEY WORDS: ACL reconstruction, antero-medial portal, trans-tibial, knee stability, soccer.

\section{Introduction}

About 75,000-100,000 anterior cruciate ligament $(A C L)$ reconstructions are performed every year in the United States ${ }^{1}$. A correct placement of bone tunnels, especially on the femoral side, is important to prevent surgical failures ${ }^{2-7}$. In fact, femoral tunnel placement influences knee kinematics ${ }^{8,9}$ and graft length ${ }^{10}$ much more than tibial tunnel. Furthermore, the femoral tunnel is more often malpositioned than tibial tunnel ${ }^{11}$, and even small differences are important on the femoral side $7,12,13$.

The femoral tunnel can be drilled in different techniques. The trans-tibial (TT) is the most popular ${ }^{14}$, however in several studies it did not provide an anatomical placement of the tunnel ${ }^{12,15}$, thus leaving a rotational instability of the knee ${ }^{9}, 16-18$. Since the first publication by Bottoni et al. ${ }^{19}$, several studies demonstrated that the antero-medial portal (AMP) technique allowed a more anatomical placement of the femoral tunnel on the lateral femoral condyle and led to better knee stability ${ }^{9}$ 16-28. In January 2009, we changed to the AMP technique in order to place the femoral tunnel closer to the anatomical ACL footprint.

The aim of this study was to retrospectively evaluate two groups of non-professional soccer players (Tegner score $=7$ points) operated on for a single bundle ACL reconstruction with hamstrings autograft, according to either one of these two techniques, and report the results in terms of stability, muscle strength, endurance, sport-specific tasks and well-validated knee scores.

\section{Methods}

From a consecutive series of single bundle ACL reconstructions, two groups of non-professional male soccer players were retrospectively selected and reviewed according to the inclusion and exclusion criteria listed in Table 1. 
Table 1. Inclusion and exclusion criteria.

\begin{tabular}{ll}
\hline Inclusion criteria & Exclusion criteria \\
\hline Active male non-professional soccer players & Other intra-articular pathologies \\
Tegner score=7 points & Previous injections in the same or contralateral knee \\
Age between 18 and 40 years old & Previous surgery on the same or contralateral knee \\
Isolated primary ACL injury treated operatively through a & \\
single bundle ACL reconstruction with hamstrings autograft & \\
Minimum 1 year after surgery & \\
A healthy contralateral knee & \\
\hline
\end{tabular}

The first group consisted of 20 patients, with a mean age at follow-up of 31 (range 22-38) years, operated on according to the TT technique ${ }^{29}$, and with a mean follow-up of $82 \pm 11$ months. The second group consisted of 23 patients, with a mean age at follow-up of 27 (range 19-38) years, operated on according to the AMP technique ${ }^{19}$, and with a mean follow-up of $27 \pm 12$ months. After surgery, patients in both groups followed the same post-operative protocol, and were prescribed the same formal physical therapy program. Patients were evaluated at follow-up with KT-1000 arthrometer (MEDmetric Corporation, San Diego, CA, USA), manual pivot shift test, isokinetic test (HumacCybex Norm, CSMI, Stoughton, MA), the incremental treadmill-running test, and athletic and sport specific tasks. The evaluation was integrated with well-validated knee functioning scales: International Knee Documentation Committee (IKDC) score ${ }^{30}$, Lysholm score ${ }^{31}$ and Knee injury and Osteoarthritis Outcome Score (KOOS) ${ }^{32}$. In all cases, the same Author performed KT-1000 measures and pivot shift tests. Anterior tibial translation was expressed as mean \pm standard deviation of the difference between operated and contralateral knee at $67 \mathrm{~N}, 134 \mathrm{~N}$, under manual maximum translation and under quadriceps contraction. Pivot shift test was classified according to the IKDC scoring system as: normal (same anterior tibial translation as the contralateral knee), nearly normal (glide), abnormal (clunk) and severely abnormal (gross instability) ${ }^{30}$. Isokinetic testing of extensor and flexor muscles of the $\mathrm{knee}^{33}$. After 20 minutes of warm-up on a stationary bike, with the patient secured on a bench by a seat belt fastened around his hips, one knee was hyperflexed and blocked on a support while the other knee was fixed to the system. The non-operated knee was attempted first. The patient trialed the machine with both knees for a few minutes before each part of the test. The test consisted in repeated active extension and flexion of the knee with the maximum strength that the patient could generate. The test was performed first at $180 \%$ sec for 20 repetitions and then at $90 \%$ sec for 4 repetitions. The more the power the patient applied to the machine, the more the resistance he received back to maintain that specific angular speed. Between the first and the second part of the test the patient rested and stretched his muscles for about 10 minutes. If the patient complained about any symptoms, the test was immediately stopped and he was invited to rest and stretch his muscles. Results were reported as the difference in percentage between the operated and the healthy knee (mean \pm standard deviation) and were classified as minimal deficit $(<10 \%)$, mild deficit $(10-25 \%)$, moderate deficit $(26-50 \%)$ severe deficit $(51-75 \%)$ and extremely severe deficit $(>75 \%)$.

The incremental treadmill-running test ${ }^{34}$ was used to evaluate aerobic and anaerobic threshold. The patient was initially asked to walk on a treadmill at 5 $\mathrm{km} / \mathrm{h}$ for 3 minutes followed by running at a speed increasing $2 \mathrm{~km} / \mathrm{h}$ every 3 minutes. After completion of each step, a blood sample was collected from the ear lobe of the patient to test lactate concentration and immediately analyzed with a sport Pro lactate analyzers YSO 1500 (Yellow Spring, CA, USA). The aerobic threshold (S2) was set at $2 \mathrm{mmol} / \mathrm{l}$ of lactate; the anaerobic threshold (S4) was set at $4 \mathrm{mmol} / \mathrm{l}$ of lactate. Results were reported as speed and heart rate at S2 and S4(mean \pm standard deviation).

A group of tests was selected for their validity as measures of fitness, strength, condition and technical skills, for their simple application in a soccer field or in a gym, and for their close relation to the normal activity of a soccer player. Strength was evaluated by a single leg squat test, single leg hop test and triple hop test for operated and non-operated limbs. Single leg squat test was performed asking the patient to stabilize his body against an examination table with one or two fingers and squat down as low as possible with one knee, while knee flexion angle was recorded with a manual goniometer. Single leg hop test was performed with the patient standing in a stepping position behind a take off line and jumping as far as possible. The best out of 3 attempts was taken for each leg, measuring the distance in centimetres from the rear most mark and the take off line. The same procedure was used for triple leg hop test, taking 3 consecutive hops on the same leg for both operated and non-operated limbs and measuring the best of 3 attempts for each side. Speed was evaluated with a shuttle run test consisting of running four times as fast as possible between two lines traced on the field 6.1 meters (20 feet) apart. Time upon the "Go" signal and crossing the last line was taken with a hand-held stop watch by the examiner and measured in units of 0.1 seconds.

For technical skills, two tests were used from FMARC (FIFA - Medical Assessment and Research Centre) test Battery that was used also by Rosch et al. ${ }^{35}$ : the speed dribbling test and the long passing test. Speed dribbling test consisted in a dribbling among 7 posts and around two blocks in a $50 \mathrm{~m}$ long field. The examiner measured total time in $0.1 \mathrm{sec}-$ 
Knee stability, athletic performance and sport-specific tasksin non-professional soccer players after ACL reconstruction: comparing trans-tibial and antero-medial portal techniques

onds units with a stop watch from the "Go" signal until the player passed through the end line and stopped with the ball under his foot. The long passing test is a passing accuracy test over a long distance. The patient was asked to pass the ball from its dead position on the line into a circle (2 meters radius, distance 36 meters) that constituted the target. The circle was in the center of a square area, $10 \times 10 \mathrm{~m}$. After a trial attempt, the player had five attempts to complete the task. Each ball that landed in the circle resulted in 3 points, 1 point if it landed in the square and no point if out of the square. The examiner measured total points amount after five attempts for each player.

Before the testing sessions, the players were informed in detail about the aims and methods used in the study methodology in order to have their consent and positive motivation in doing the tests.

\section{Statistical analysis}

All measurements were reported as mean \pm standard deviation. An unpaired t-test was used to compare both groups with respect to objective outcomes. For all statistical tests, the alpha level was set at 0.05. Statistical analyses were performedwith SPSS v.15.0 (SPSS Inc., an IBM Company, Chicago, IL, USA). The study meets the ethical standards of the journal ${ }^{36}$.

\section{Results}

Results at KT-1000 evaluation are reported in Table 2. The two groups obtained similar results without statis- tically significant differences ( $p>0.05$ ). At manual pivot shift test, a glide was present in all patients of TT group. On the other hand, none of the patients in AMP group showed a positive pivot shift test $(p<0.05)$.

Results of isokinetic test are reported in Table 3. Both groups showed only a minimal deficit of flexor and extensor muscles strength and a low flexor/extensor muscles ratio. In the TT group, the flexor deficit at $90 \%$ sec was mild $(-10.5 \%)$. Both groups showed comparable results of isokinetic testing, without any statistically significant difference ( $p>0.05)$.

Results of the incremental treadmill-running test are reported in Table 4. Both groups ran at comparable speed at S2 and S4 without any statistically significant difference $(p>0.05)$, but patients in AMP group showed significantly higher heart rates both at S2 and S4 ( $p=0.001$ and 0.02 respectively).

In the TT group the mean knee flexion angles during single leg squat test were $77^{\circ} \pm 5^{\circ}$ in the operated knee and $89^{\circ} \pm 7^{\circ}$ in the non-operated knee ( $\left.p=0.001\right)$. The mean jumped distance at the single leg hop was $122 \pm 30 \mathrm{~cm}$ in the operated knee and $134 \pm 24 \mathrm{~cm}$ in the contralateral knee ( $p>0.05)$. The mean jumped distance at the triple leg hop test was $339 \pm 76 \mathrm{~cm}$ in the operated knee and $420 \pm 63 \mathrm{~cm}$ in the contralateral knee ( $p>0.05)$. In the AMP group the mean knee flexion angles during squat test were $83^{\circ} \pm 18^{\circ}$ in the operated knee and $89^{\circ} \pm 14^{\circ}$ in the non-operated knee ( $p>0.05$ ). The mean jumped distance at the single leg hop was $126 \pm 34 \mathrm{~cm}$ in the operated knee and $140 \pm 37 \mathrm{~cm}$ in the contralateral knee $(p>0.05)$. The mean jumped distance at the triple leg hop test was $387 \pm 104 \mathrm{~cm}$ in the operated knee and $409 \pm 118 \mathrm{~cm}$ in the contralateral knee $(p>0.05)$. The mean times at

Table 2. Results at KT-1000 arthrometer (mean \pm standard deviation).

\begin{tabular}{llll}
\hline Strength & TT group & AMP group & $P$ \\
\hline $67 \mathrm{~N}$ & $1.3 \pm 0.5 \mathrm{~mm}$ & $0.8 \pm 0.4 \mathrm{~mm}$ & $>0.05$ \\
$134 \mathrm{~N}$ & $1.6 \pm 0.8 \mathrm{~mm}$ & $1.0 \pm 1 \mathrm{~mm}$ & $>0.05$ \\
Manual maximum & $3.2 \pm 2.4 \mathrm{~mm}$ & $2.3 \pm 2.4 \mathrm{~mm}$ & $>0.05$ \\
Quadriceps contraction & $1.0 \pm 1.3 \mathrm{~mm}$ & $0.7 \pm 1.4 \mathrm{~mm}$ & $>0.05$ \\
\hline
\end{tabular}

Table 3. Results of isokinetic test at $180 \% \mathrm{sec}$ and $90 \% \mathrm{sec}$ (mean \pm standard deviation).

\begin{tabular}{llll}
\hline & TT group & AMP group & $P$ \\
\hline Difference in extensor strength at $180 \% / \mathrm{sec}$ & $-7.9 \pm 16.5 \%$ & $-5.8 \pm 18.2 \%$ & $>0.05$ \\
Difference in flexor strength at $180 \% \mathrm{sec}$ & $-1.0 \pm 9.4 \%$ & $-9.7 \pm 20 \%$ & $>0.05$ \\
Flexor/extensor ratio at $180 \% \mathrm{sec}$ & $61.9 \pm 8.4$ & $58.5 \pm 5.9$ & $>0.05$ \\
Difference in extensor strength at $90 \% \mathrm{sec}$ & $-10.5 \pm 10.6 \%$ & $-9.5 \pm 14.3 \%$ & $>0.05$ \\
Difference in flexor strength at $90 \% / \mathrm{sec}$ & $-6.6 \pm 7.1 \%$ & $-3.5 \pm 14.5 \%$ & $>0.05$ \\
Flexor/extensor ratio at $90 \% \mathrm{sec}$ & $58.8 \pm 9.5$ & $56.6 \pm 2.6$ & $>0.05$ \\
\hline
\end{tabular}

Table 4. Results of the incremental treadmill-running test (mean \pm standard deviation).

\begin{tabular}{llll}
\hline & TT group & AMP group & $P$ \\
\hline S2 speed & $8.2 \pm 1.4 \mathrm{~km} / \mathrm{h}$ & $8.7 \pm 2.3 \mathrm{~km} / \mathrm{h}$ & $>0.05$ \\
S2 heart rate & $153 \pm 6.2 \mathrm{bpm}$ & $165 \pm 7.9 \mathrm{bpm}$ & 0.001 \\
S4 speed & $10.5 \pm 1.3 \mathrm{~km} / \mathrm{h}$ & $11.3 \pm 2.8 \mathrm{~km} / \mathrm{h}$ & $>0.05$ \\
S4 heart rate & $175 \pm 9.9 \mathrm{bpm}$ & $185 \pm 7.6 \mathrm{bpm}$ & 0.02 \\
\hline
\end{tabular}


shuttle test were $7.5 \pm 0.4 \mathrm{sec}$ and $7.6 \pm 0.2 \mathrm{sec}$ for TT and AMP groups, respectively. The mean times at speed dribbling tests were $27.1 \pm 3.2 \mathrm{sec}$ and $25.0 \pm 1.1$ sec for TT and AMP groups, respectively. The mean scores at long passing test were $2.6 \pm 0.9$ and $2.3 \pm 2.0$ points for TT and AMP groups, respectively. Comparing TT and AMP groups, there were no statistically significant differences in single leg squat, single leg hop, triple leg hop, shuttle, speed dribbling and long passing tests ( $p>0.05)$.

The mean IKDC scores were $73.14 \pm 13.20$ and $78.53 \pm 14.96$ in the TT and AMP groups, respectively $(p>0.05)$. The mean Lysholm Knee scores were $80.90 \pm 12.30$ and $84.87 \pm 14.43$ in the TT and AMP groups, respectively ( $p>0.05)$. The mean KOOS were $82.38 \pm 7.74$ and $87.94 \pm 9.40$ in the TT and AMP groups, respectively $(p=0.04)$.

\section{Discussion}

The anatomical results of TT and AMP techniques, in terms of femoral tunnel placement, are still controversial. Several studies showed that independent femoral tunnel placement led to a more anatomical position of the ACL on both coronal and sagittal planes $8,9,20-24$. Other Authors found no differences in terms of tunnel placement with the two techniques ${ }^{37-39}$. Rue demonstrated that an anatomical placement of the femoral tunnel could be obtained also with a TT approach ${ }^{40}$ and Musahl et al. ${ }^{5}$ demonstrated that neither tunnel placement between the anatomical origin of the $A C L$ and the most isometric point fully restored knee kinematics, but an anatomic graft placement resulted in a kinematics similar to the normal knee joint.

Also, clinical results of TT and AMP techniques are controversial. A review of the literature ${ }^{41}$ showed that patients in which the femoral tunnel was drilled with the AMP technique begin running significantly earlier, had significantly greater range of motion and anteroposterior knee stability at short term follow-up, but these differences were not evident at medium and long term follow-ups. In contrast, patients with a TT reconstruction had greater activity level at medium and long-term follow-up and lower failure rate of the graft. The Authors found no differences in terms of clinical function between the two groups and concluded that there is no evidence that one technique is superior to the other. However, as observed by the Authors, their study is based on indirect comparison of data from non-homogeneous studies and the results should be considered with caution.

The same group ${ }^{42}$ published the first study that, to the best of our knowledge, directly compared the clinical results of two groups of patients operated on for an $A C L$ reconstruction in which the femoral tunnel was drilled either with a TT or AMP technique. They concluded that the AMP group had significantly improved anterior-posterior and rotational knee stability, IKDC scores, and recovery time from surgery; but no differences were found for VAS for satisfaction with surgery, Lysholm, Tegner, and SF-12 between both groups.
Subsequently, the data from the Danish register were published $^{43}$, reporting an increased risk for revision, positive pivot shift and antero-posterior instability in a large group of patients associated with AMP technique. As in every register study, many data are missing about surgical technique, post-operative protocols, fixation devices, surgeons' skills and learning curve, and selection of the patients. Therefore the results should be considered with caution.

Franceschi et al. ${ }^{44}$, in their retrospective analysis of prospectively collected data on 88 athletes, at a minimum follow-up of 5 years, reported non-significant better rotational stability and anterior-translation in the AMP group, without significant differences in IKDC and Lysholm scores or in degenerative changes between the two groups at a minimum follow-up of 5 years.

Koutras et al. ${ }^{45}$ reported that the AMP group had significantly better Lysholm scores at 3 months and better performance in the timed lateral movement functional tests at 3 and 6 months. All other analyzed comparisons were not significantly different between the two groups.

Duffee et al. ${ }^{46}$ reported in a large cohort of 380 patients, at 6 years follow-up, a higher reoperation rate in the ipsilateral knee in the TT group compared to AMP group. Reoperations were done for many different reasons including, but not limited to, anterior debridement, revision ACL surgery, meniscal surgery and cartilage procedures. The Authors did not report about the clinical results of the patients at follow-up because their data were extracted from the MOON database and not from direct evaluation of the patients.

Wang et al. ${ }^{47}$ in their gait analysis study on 24 patients, reported that the AMP technique better restored the anterior-posterior translation during the swing phase and femoral external rotation at midstance compared to the TT technique. On the other hand, the AMP technique was correlated with an extension loss of 5 degrees during the late stance phase.

The results of the present study showed that only rotational stability of the knee was better restored in AMP group. There were no statistically significant differences in the outcomes at isokinetic tests, incremental treadmill test, strength tests (single leg squat test, single leg hop test, triple leg hop test), speed tests (shuttle test and dribbling test), long passing test, nor functional scores (Lysholm, IKDC). Only KOOS was significantly higher in AMP group $(p=0.04)$. The results of the isokinetic test indicated a low flexor/extensor ratio in both groups, at $180 \% \mathrm{sec}$ and $90 \% \mathrm{sec}$, that may be associated with an incomplete physical therapy program after surgery with patients returning too early to practice, or to an insufficient strengthening program with the soccer team. The results of the incremental treadmill-running test indicated in both groups a poor athletic condition, perhaps related to inadequate aerobic training with the soccer teams during practices.

Strengths of the study. Patients were evaluated in terms of multiple variables, strength, endurance, power, speed, technical skills and functional scores. Strict 
Knee stability, athletic performance and sport-specific tasksin non-professional soccer players after ACL reconstruction: comparing trans-tibial and antero-medial portal techniques

inclusion and exclusion criteria were used to select patients.

Limitations of the study. This is a retrospective study. Patients were not randomized, nor were they matched for pre-operative variables. The 2 groups have different follow-up times. Rotational instability was evaluated with a manual pivot shift test that can be criticized for being very subjective but it is adopted in the daily clinical practice by many orthopedists.

The results of the published studies changed the trends of ACL reconstruction over time. In fact, while $85 \%$ of the members of the American Academy of Orthopaedic Surgeons in 2009 used to dill the femoral tunnel with a TT approach and 15\% through the $\mathrm{AMP}^{14}$, a more recent international survey published in 2013 reported that the most preferred surgical approach was the AMP technique (68\% of responders), followed by the TT $(31 \%)$ and open (1\%), with no significant differences based on geographic origin, specialty or seniority ${ }^{48}$. More clinical studies are needed in the future to better evaluate the difference between TT and AMP techniques for drilling the femoral tunnel during $A C L$ reconstruction and to assess whether the better rotational stability achieved through the AMP technique, is related to better or quicker return to sports, that is very important for athletes.

\section{Conclusion}

The group of patients in which femoral tunnel was drilled through the AMP obtained better results atmanual pivot shift test and KOOS, but lower flexion angles at single leg squat test. All the other analyzed variables were not significantly different at follow-up between the two groups. The better rotational stability of the knee achieved drilling the femoral tunnel through the AMP did not lead to significantly better clinical and functional results in our patients.

\section{References}

1. Walsh MP, Wijdicks CA, Armitage BM, Westerhaus BD, Parker JB, LaPrade RF. The 1:1 versus the 2:2 tunnel-drilling technique: optimization of fixation strength and stiffness in an allinside double-bundle anterior cruciate ligament reconstruction-a biomechanical study. Am J Sports Med. 2009;37:15391547.

2. Getelman MH, Friedman MJ. Revision anterior cruciate ligament reconstruction surgery. J Am Acad Orthop Surg. 1999;7: 189-198.

3. Johnson DL, Swenson TM, Irrgang JJ, Fu FH, Harner CD. Revision anterior cruciate ligament surgery: experience from Pittsburgh. Clin Orthop Relat Res. 1996;325:100-109.

4. Lind $M$, Menhert $F$, Pedersen $A B$. The first results from the Danish ACL reconstruction registry: epidemiologic and 2 year follow-up results from 5,818 knee ligament reconstructions. Knee Surg Sports Traumatol Arthrosc. 2009;17:117-124.

5. Musahl V, Plakseychuk A, VanScyoc A, Sasaki T, Debski RE, McMahon PJ, Fu FH. Varying femoral tunnels between the anatomical footprint and isometric positions: effect on kinematics of the anterior cruciate ligament-reconstructed knee. Am J Sports Med. 2005;33:712-718.
6. Wetzler MJ, Getelman MH, Friedman MJ, Bartolozzi AR. Revision anterior cruciate ligament surgery: etiology of failures. Operative Techiques Sport Med. 1998;6:64-70.

7. Zavras TD, Race A, Bull AM, Amis AA. A comparative study of 'isometric' points for anterior cruciate ligament graft attachment. Knee Surg Sports Traumatol Arthrosc. 2001;9:28-33.

8. Loh JC, Fukuda Y, Tsuda E, Steadman RJ, Fu FH, Woo SL. Knee stability and graft function following anterior cruciate ligament reconstruction: Comparison between 11 o'clock and 10 o'clock femoral tunnel placement. 2002 Richard O'Connor Award paper. Arthroscopy. 2003;19:297-304.

9. Scopp JM, Jasper LE, Belkoff SM, Moorman CT 3rd. The effect of oblique femoral tunnel placement on rotational constraint of the knee reconstructed using patellar tendon autografts. Arthroscopy. 2004;20:294-299.

10. Muneta T, Yamamoto H, Sakai H, Ishibashi T, Furuya K. Relationship between changes in length and force in in vitro reconstructed anterior cruciate ligament. Am J Sports Med. 1993; 21:299-304.

11. Topliss $C$, Webb J. An audit of tunnel position in anterior cruciate ligament reconstruction. Knee. 2001;8:59-63.

12. Aglietti $P$, Buzzi R, D'Andria S, Zaccherotti G. Long-term study of anterior cruciate ligament reconstruction for chronic instability using the central one-third patellar tendon and a lateral extraarticulartenodesis. Am J Sports Med. 1992;20:38-45.

13. Khalfayan EE, Sharkey PF, Alexander AH, Bruckner JD, Bynum EB. The relationship between tunnel placement and clinical results after anterior cruciate ligament reconstruction. Am J Sports Med. 1996;24:335-341.

14. Duquin TR, Wind WM, Fineberg MS, Smolinski RJ, Buyea $\mathrm{CM}$. Current trends in anterior cruciate ligament reconstruction. J Knee Surg. 2009;22:7-12.

15. Grøntvedt T, Pena F, Engebretsen L. Accuracy of femoral tunnel placement and resulting graft force using one- or two-incision drill guides. A cadaver study on ten paired knees. Arthroscopy. 1996;12:187-192.

16. Howell SM, Gittins ME, Gottlieb JE, Traina SM, Zoellner TM The relationship between the angle of the tibial tunnel in the coronal plane and loss of flexion and anterior laxity after anterior cruciate ligament reconstruction. Am J Sports Med. 2001;29:567-574.

17. Lee MC, Seong SC, Lee S, Chang CB, Park YK, Jo H, Kim CH Vertical femoral tunnel placement results in rotational knee laxity after anterior cruciate ligament reconstruction. Arthroscopy. 2007;23:771-778.

18. Yamamoto $Y$, Hsu WH, Woo SL, Van Scyoc AH, Takakura $Y$, Debski RE. Knee stability and graft function after anterior cruciate ligament reconstruction: a comparison of a lateral and an anatomical femoral tunnel placement. Am J Sports Med. 2004;32:1825-1832.

19. Bottoni CR, Rooney RC, Harpstrite JK, Kan DM. Ensuring accurate femoral guide pin placement in anterior cruciate ligament reconstruction. Am J Orthop (Belle Mead NJ). 1998;27: 764-766.

20. Cha PS, Chhabra A, Harner CD. Single-bundle anterior cruciate ligament reconstruction using the medial portal technique. Oper Tech Orthop. 2005;15:85-89.

21. Dargel J, Schmidt-Wiethoff R, Fischer S, Mader K, Koebke J, Schneider T. Femoral bone tunnel placement using the transtibial tunnel or the anteromedial portal in ACL reconstruction: a radiographic evaluation. Knee Surg Sports Traumatol Arthrosc. 2009:17:220-227.

22. Gavriilidis I, Motsis EK, Pakos EE, Georgoulis AD, Mitsionis G, Xenakis TA. Transtibial versus anteromedial portal of the femoral tunnel in ACL reconstruction: a cadaveric study. Knee. 2008;15:364-367.

23. Hantes ME, Zachos VC, Liantsis A, Venouziou A, Karantanas $\mathrm{AH}$, Malizos KN. Differences in graft orientation using the 
transtibial and anteromedial portal technique in anterior cruciate ligament reconstruction: a magnetic resonance imaging study. Knee Surg Sports Traumatol Arthrosc. 2009;17:880886.

24. Harner CD, Honkamp NJ, Ranawat AS. Anteromedial portal technique for creating the anterior cruciate ligament femoral tunnel. Arthroscopy. 2008;24:113-115.

25. Noh JH, Roh YH, Yang BG, Yi SR, Lee SY. Femoral tunnel position on conventional magnetic resonance imaging after anterior cruciate ligament reconstruction in young men: transtibial technique versus anteromedial portal technique. Arthroscopy. 2013;29:882-890.

26. Robert HE, Bouguennec N, Vogeli D, Berton E, Bowen M. Coverage of the anterior cruciate ligament femoral footprint using 3 different approaches in single-bundle reconstruction: a cadaveric study analyzed by 3-dimensional computed tomography. Am J Sports Med. 2013;41:2375-2383.

27. Shin YS, Ro KH, Lee JH, Lee DH. Location of the femoral tunnel aperture in single-bundle anterior cruciate ligament reconstruction: comparison of the transtibial, anteromedial portal, and outside-in techniques. Am J Sports Med. 2013;41:25332539.

28. Tudisco C, Bisicchia S. Drilling the femoral tunnel during ACL reconstruction: transtibial versus anteromedial portal techniques. Orthopedics. 2012;35:e1166-1172.

29. Hardin GT, Bach BR, Bush-Joseph CA, Farr J. Endoscopic single-incision anterior cruciate ligament reconstruction using patellar tendon autograft. Am J Knee Surg. 1992;5:144-155.

30. Hefti F, Muller W, Jakob RP, Stäubli HU. Evaluation of knee ligament injuries with the IKDC form. Knee Surg Sports TraumatolArthrosc. 1993;1:226-234.

31. Lysholm J, Gillquist J. Evaluation of knee ligament surgery results with special emphasis on use of a scoring scale. Am J Sports Med. 1982;10:150-154.

32. Roos EM, Roos HP, Lohmander LS, Ekdahl C, Beynnon BD. Knee Injury and Osteoarthritis Outcome Score (KOOS) - development of a self-administered outcome measure. J Orthop Sports Phys Ther. 1998;28:88-96.

33. Almekinders LC, Oman J. Isokinetic Muscle Testing: Is It Clinically Useful? J Am Acad Orthop Surg. 1994;2:221-225.

34. Foster C, Fitzgerald DJ, Spatz P. Stability of the blood lactateheart rate relationship in competitive athletes. Med Sci Sports Exerc. 1999;31:578-582.

35. Rosch D, Hodgson R, Peterson L, Graf-Baumann T, Junge A, Chomiak J, Dvorak J. Assessment and evaluation of football performance. Am J Sports Med. 2000;28(Suppl 5):S29-39.

36. Padulo J, Oliva F, Frizziero A, Maffulli N. Muscles, Ligaments and Tendons Journal. Basic principles and recommendations in clinical and field science. MLTJ. 2013;4:250-252.

37. Albuquerque RF, Amatuzzi MM, Pacheco AP, Angelini FJ, Campos O Jr. Positioning of the femoral tunnel for arthroscop- ic reconstruction of the anterior cruciate ligament: comparative study of 2 techniques. Clinics (Sao Paulo). 2007;62:613-618.

38. Bedi A, Raphael B, Maderazo A, Pavlov H, Williams RJ III. Transtibial versus anteromedial portal drilling for anterior cruciate ligament reconstruction: a cadaveric study of femoral tunnel length and obliquity. Arthroscopy. 2010;26:342-350.

39. Giron F, Buzzi R, Aglietti P. Femoral tunnel position in anterior cruciate ligament reconstruction using three techniques. A cadaver study. Arthroscopy. 1999;15:750-756.

40. Rue JP, Ghodadra N, Bach BR Jr. Femoral tunnel placement in single-bundle anterior cruciate ligament reconstruction: a cadaveric study relating transtibial lateralized femoral tunnel position to the anteromedial and posterolateral bundle femoral origins of the anterior cruciate ligament. Am J Sports Med. 2008;36:73-79.

41. Alentorn-Geli E, Lajara F, Samitier G, Cugat R. The transtibial versus the anteromedial portal technique in the arthroscopic bone-patellar tendon-bone anterior cruciate ligament reconstruction. Knee Surg Sports Traumatol Arthrosc. 2010;18: 1013-1037.

42. Alentorn-Geli E, Samitier G, Alvarez P, Steinbacher G, Cugat $R$. Anteromedial portal versus transtibial drilling techniques in ACL reconstruction: a blinded cross-sectional study at two- to five-year follow-up. Int Orthop. 2010;34:747-754.

43. Rahr-Wagner L, ThillemannTM, Pedersen AB, Lind MC. Increased risk of revision after anteromedial compared with transtibial drilling of the femoral tunnel during primary anterior cruciate ligament reconstruction: results from the Danish Knee Ligament Reconstruction Register. Arthroscopy. 2013;29:98105.

44. Franceschi F, Papalia R, Rizzello G, Del Buono A, Maffulli N, Denaro V. Anteromedial portal versus transtibial drilling techniques in anterior cruciate ligament reconstruction: any clinical relevance? A retrospective comparative study. Arthroscopy. 2013;29:1330-1337.

45. Koutras G, Papadopoulos P, Terzidis IP, Gigis I, Pappas E.Short-term functional and clinical outcomes after ACL reconstruction with hamstrings autograft: transtibial versus anteromedial portal technique. Knee Surg Sports Traumatol Arthrosc. 2013;21:1904-1909.

46. Duffee A, Magnussen RA, Pedroza AD, Flanigan DC, MOON Group, Kaeding CC. Transtibial ACL femoral tunnel preparation increases odds of repeat ipsilateral knee surgery. J Bone Joint Surg Am. 2013;95:2035-2042.

47. Wang H, Fleischli JE, Zheng NN. Transtibial versus anteromedial portal technique in single-bundle anterior cruciate ligament reconstruction: outcomes of knee joint kinematics during walking. Am J Sports Med. 2013;41:1847-1856.

48. Chechik O, Amar E, Khashan M, Lador R, Eyal G, Gold A. An international survey on anterior cruciate ligament reconstruction practices. Int Orthop. 2013;37(2):201-206. 